\title{
Stimulation of in vitro bone formation by canine prostate cancer
}

\author{
Shiyu Yuan ${ }^{1,2}$, Noriko Kantake ${ }^{1}$, Daniel E. Hellmann', Said M. Elshafae ${ }^{3}$, Thomas J. Rosol ${ }^{1,2,4}$ \\ 'Department of Biomedical Sciences, Heritage College of Osteopathic Medicine, Ohio University, Athens, OH 45701, USA. \\ ${ }^{2}$ Molecular and Cellular Biology Program, Ohio University, Athens, OH 45701, USA. \\ ${ }^{3}$ Faculty of Veterinary Medicine, Benha University, Tukh, Qalyobiya 13736, Egypt. \\ ${ }^{4}$ Ohio Musculoskeletal and Neurological Institute, Ohio University, Athens, OH 45701, USA
}

Correspondence to: Dr. Thomas J. Rosol, Department of Biomedical Sciences, Heritage College of Osteopathic Medicine, Ohio University, 225 Irvine Hall, Athens, OH 45701, USA. E-mail: rosolt@ohio.edu

How to cite this article: Yuan S, Kantake N, Hellmann DE, Elshafae SM, Rosol TJ. Stimulation of in vitro bone formation by canine prostate cancer. J Cancer Metastasis Treat 2021;7:37. https://dx.doi.org/10.20517/2394-4722.2021.41

Received: 19 Feb 2021 First Decision: 22 Mar 2021 Revised: 6 Apr 2021 Accepted: 15 Apr 2021 First online:: 8 Jun 2021

Academic Editor: RE Coleman Copy Editor: Xi-Jun Chen Production Editor: Xi-Jun Chen

\begin{abstract}
Aim: Patients with prostate cancer frequently develop osteoblastic bone metastases. Canine models are important because dogs are the only mammal to develop spontaneous prostate cancer with osteoblastic bone metastases similar to men. The mechanism by which prostate cancer induces bone formation is unclear; however, it depends on the complex interaction between prostate cancer cells and bone microenvironment. This study investigated the effects of three canine prostate cancer cell lines (Ace-1, LuMa, and Probasco) on bone formation and resorption in vitro.
\end{abstract}

Methods: Mouse calvaria were treated with conditioned medium (CM) from cell lines. Calvaria were evaluated by histology, fluorescent calcein uptake at sites of bone mineralization, medium calcium assay, and alkaline phosphatase activity. The expression of bone-related genes was measured using quantitative reverse transcriptionPCR.

Results: A novel calcein uptake assay was developed to measure bone formation and mineralization in vitro. Ace-1 CM induced predominantly bone resorption in calvaria, while Probasco CM induced marked bone formation, mineralization, and healing of calvaria defects. The expression of osteoblast-related genes in calvaria showed that Probasco CM stimulated the maturation and differentiation of osteoblasts and inhibited osteoclastogenesis. Both bone modeling and remodeling were involved in Probasco CM-induced bone formation and mineralization by inhibiting remodeling with zoledronic acid. Inhibition of WNT activity by DKK-1 decreased the osteoblastic activity 
of Probasco cells.

Conclusion: Probasco cells induced bone formation and mineralization in vitro that depended on the WNT signaling pathway. Probasco cells will serve as a valuable model for studying the mechanisms of osteoblastic bone metastasis in prostate cancer.

Keywords: Bone, canine, dog, metastasis, osteoblast, prostate cancer, zoledronic acid, DKK-1

\section{INTRODUCTION}

Prostate cancer ( $\mathrm{PCa}$ ) metastasizes to bone and induces "osteoblastic" metastases that are characterized by increased woven bone formation initially on endosteal bone surfaces and can eventually extend beyond the normal limits of the bone ${ }^{[1]}$. This results in a metastasis composed of prostate cancer tissue and dense bone that can be visualized by radiography. Osteoblastic metastases are often associated with bone pain, impaired mobility, and spinal cord compression that severely affect the patients' quality of life ${ }^{[2]}$.

Dogs have a lobular prostate gland that is similar to men's in size and intact male dogs develop benign prostatic hyperplasia as they age similar to $\mathrm{men}^{[3]}$. Dogs are the only animal that develops spontaneous prostate cancer with metastasis to bone with the formation of osteoblastic metastases ${ }^{[4]}$. Most metastatic prostate cancers in dogs are androgen-independent and represent a valuable animal model for late-stage androgen-resistant prostate cancer as it occurs in men $^{[5]}$.

Our laboratory has developed multiple prostate cancer cell lines from spontaneous prostate cancer in dogs. This investigation utilized three different cell lines $\left(\right.$ Ace $-{ }^{[6]}$, Probasco ${ }^{[7]}$, and $\mathrm{LuMa}^{[8]}$ ) that vary in their in vivo phenotype in athymic mice. All metastasize to bone and induce different degrees of osteoblastic bone formation and osteoclastic bone resorption.

Several factors have been implicated as contributing to osteoblastic metastasis including WNT proteins ${ }^{[0]}$. WNT proteins are soluble glycoproteins that bind to receptor complexes composed of LRP5/6 and Frizzled protein $\mathrm{s}^{[10]}$. Although the function of WNTs in bone development and homeostasis is well elucidated, the role of WNT signaling in the interaction between PCa cells and bone microenvironment is complicated and remains poorly understood. Previously we have shown that inhibition of bone formation by prostate cancer by blocking WNT action with DKK-1 resulted in increased bone metastases and more rapid tumor growth $^{[11]}$. This suggests that bone formation in osteoblastic metastases plays an important role in the regulation of cancer progression.

Since the pathogenesis of bone formation in prostate cancer bone metastases is unknown, we developed a novel in vitro model system for measurement of bone formation. In vitro cultures of mouse calvaria is a time-honored system for culture of bone tissue and is especially useful for investigating in vitro osteoclastic bone resorption ${ }^{[12]}$. Measurement of in vitro bone formation using calvaria has been less utilized because it is more technically challenging. There are few reports of bone formation induction in vitro in calvaria. We have optimized the use of calcein, a fluorescent biomarker of bone mineralization, to permit measurement of bone formation in vitro ${ }^{[13]}$.

The overall goal of this investigation was to collect conditioned medium (CM) from cultured canine prostate cancer cell lines and measure their effect on bone formation and resorption and bone healing in vitro. In addition, the mRNA expression for bone-specific proteins and candidates for induction of bone 
formation were measured. DKK-1 was used as a broad inhibitor of WNT signaling to determine the role of the WNT pathway on bone formation in vitro.

\section{METHODS}

Cell lines, conditioned medium, and chemicals

Four canine prostatic cancer cell lines were used in this study including Ace- $1^{[6]}$, Probasco ${ }^{[7]}$, Probasco+DKK-1 (Probasco cells transduced with human DKK-1), and $\mathrm{LuMa}^{[8]}$ (all cells were established by our laboratory). Cells were cultured in DMEM/F12 (Gibco, Carlsbad, CA) containing 10\% fetal bovine serum (FBS), 1\% penicillin/streptomycin and $100 \mu \mathrm{g} / \mathrm{mL}$ Normocin (Invivogen, San Diego, CA). All cells were serially passage using trypsinization and maintained at $37{ }^{\circ} \mathrm{C}$ and $5 \% \mathrm{CO}_{2}$ in a humidified atmosphere. CM was prepared by incubating near confluent cells in T75 flask for $24 \mathrm{~h}$ with serum-free BGJb culture medium (Gibco) with $0.1 \%$ bovine serum albumin (BSA) fraction V (Sigma-Aldrich Corp, St Louis, Missouri) and $100 \mu \mathrm{g} / \mathrm{mL}$ Normocin. CM was stored in aliquots at $-80{ }^{\circ} \mathrm{C}$ until used. ZOL (zoledronic acid, Zometa $^{\mathrm{TM}}$, Novartis) was purchased from the James Cancer Center at Ohio State University.

\section{In vitro cultures of mouse calvaria}

Calvaria of 4- to 7-day-old CD1 pups were aseptically removed and dissected from surrounding soft tissues, cut in half, and incubated in $1 \mathrm{~mL}$ serum-free BGJb medium or conditioned medium for 2-6 days at $37{ }^{\circ} \mathrm{C}$ and $5 \% \mathrm{CO}_{2}$ in a humidified atmosphere. Medium was changed every other day in both control (BGJb + calvaria only) and treatment groups (50\% BGJb $+50 \% \mathrm{CM}+$ calvaria) until the end of the experiment.

\section{Wound healing of calvaria defect}

An in vitro bone healing model was used to evaluate the induction of new bone formation by Probasco conditioned media. A $1.5 \mathrm{~mm}$ bone disk was punched and removed from the parietal bone on both sides in each calvaria using biopsy punches (Braintree Scientific, Braintree, MA) to create artificial defects. Calvaria with defects were treated with CM or control (plain media) for 6 days. The calvarial defects were imaged at day 6 using a dissecting microscope (Nikon SMZ-U) and the repaired areas were quantified using imageJ (NIH, version 2.0).

\section{Calcein uptake and bone formation with mineralization}

A novel in vitro bone formation and mineralization assay was developed. Each half calvarium was cultured in BGJb medium or 50\% conditioned medium for 6 days. Calcein (Sigma-Aldich), a green, fluorescent dye with excitation and emission wavelengths of $495 / 515 \mathrm{~nm}$ that binds to calcium at sites of bone formation and mineralization, was added to the cultures $(0.4 \mu \mathrm{g} / \mathrm{mL})$ at day 4 . At the end of the experiment, calvaria were fixed in $10 \%$ neutral-buffered formalin at room temperature for $1.5 \mathrm{~h}$. A bone disk $(1.5 \mathrm{~mm})$ was removed from each side of the fixed mouse calvaria using dermatology biopsy punches and then mounted on glass slides. Newly mineralized bone labelled by calcein was detected and imaged using a Nikon E600 fluorescent microscope with a digital camera. Total fluorescence intensity was quantified using imageJ $(\mathrm{NIH}$, version 2.0) to measure the amount of new bone formation.

\section{Histopathology}

Fixed calvaria were decalcified (10\% EDTA, $\mathrm{pH}$ 7.4, for $24 \mathrm{~h}$ at $4{ }^{\circ} \mathrm{C}$ ), paraffin-embedded, $4 \mu \mathrm{m}$ sections cut, mounted on glass slides, and stained with hematoxylin and eosin (H\&E) for examining the changes of calvaria thickness. Undecalcified calcein-treated calvaria were embedded in OCT, $10 \mathrm{~mm}$ sections cut on a cryomicrotome, and mounted for viewing calcein uptake. 


\section{Alkaline phosphatase activity assay}

$3.5 \mathrm{~mm}$ calvaria discs (after 6 days of incubation with BGJb or CM) were extracted in $500 \mu \mathrm{L} 0.01 \%$ triton X100 (containing $0.02 \%$ azide) for $72 \mathrm{~h}$ at $4{ }^{\circ} \mathrm{C}$, and extracted protein was collected. Alkaline phosphatase (ALP) activity was measured by the time-dependent formation of p-nitrophenolate (pNP) from ALP substrate solution (pNPP) at room temperature for $1 \mathrm{~h}^{[14]}$. Typically, $10 \mu \mathrm{L}$ of extract was incubated in a total of volume of $100 \mu \mathrm{L}$ containing $4.7 \mathrm{mM}$ pNPP, $1 \mathrm{mM} \mathrm{MgCl}_{2}$ and $1.02 \mathrm{M}$ diethanolamine buffer, $\mathrm{pH} 9.8$, in a 96-place microtiter plate. Each sample was measured in duplicate. The reactions were initiated by addition of substrate, and the time-dependent increase in absorbance at $405 \mathrm{~nm}$ was measured every 5 min (Synergy ${ }^{\mathrm{TM}}$ HTX Multi-Mode Microplate Reader). ALP activity was calculated as $\mathrm{pNP}$ produced/min using a standard curve.

\section{Calcium concentration in calvaria medium}

Calvaria culture medium supernatant was collected on day 1 and day 2, and the total medium calcium was measured as described by Lorent $\mathrm{z}^{[15]}$.

\section{Dickkopf-1 enzyme-linked immunosorbent assay}

DKK-1 levels in conditioned medium were measured using the DuoSet Human DKK1 enzyme-linked immunosorbent assay (ELISA) Kit, as recommended by the manufacturer (R\&D Systems, Minneapolis, $\mathrm{MN})$. The lowest standard of the assay was $62.5 \mathrm{pg} / \mathrm{mL}$.

\section{RNA extraction and quantitative reverse-transcription polymerase chain reaction}

Total RNA was extracted from calvaria using RNeasy mini kit (Qiagen). Then total RNA ( $0.5 \mu \mathrm{g})$ was reverse transcribed in cDNA using the Superscript II First Strand cDNA synthesis kit (Invitrogen). Quantitative RT polymerase chain reaction (PCR) was performed for the reference gene, ubiquitin $\mathrm{C}(U B C)$, osteoblast-related genes including runt-related transcription factor 2 (RUNX2), Osterix (OSX), Alkaline phosphatase $(A L P L)$, receptor activator of nuclear factor kappa-B ligand (RANKL), osteoprotegerin $(O P G)$, osteocalcin $(O C N)$, integrin-binding sialoprotein $(I B S P)$, parathyroid hormone 1 receptor $(P T H 1 R)$, osteomodulin $(O M D)$, and osteonectin $(O N)$, and osteoclast-related genes including matrix metalloproteinase $9(M M P 9)$ and cathepsin $\mathrm{K}(C T S K)$. The primers are listed in Table 1 . The thermal cycle conditions were: $95{ }^{\circ} \mathrm{C}, 3 \mathrm{~min} ;\left(95{ }^{\circ} \mathrm{C}, 10 \mathrm{~s} ; 55{ }^{\circ} \mathrm{C}, 30 \mathrm{~s} ; 75{ }^{\circ} \mathrm{C}, 30 \mathrm{~s}\right) \times 39 ; 95{ }^{\circ} \mathrm{C}, 1 \mathrm{~min} ; 55{ }^{\circ} \mathrm{C}, 1 \mathrm{~min}$. The expression of targeted genes was normalized by $U B C$ and quantified using the $2^{-\Delta \Delta \mathrm{Ct}}$ method.

\section{Statistical analysis}

All data were displayed as mean \pm standard deviation. Paired student's $t$-test was used for bivariate analyses. ANOVA was used to analyze multivariate comparisons. Data with $P$ values $\leq 0.05$ were considered statistically significant. Statistical calculation was performed using GraphPad Prism version 9 (La Jolla, CA).

\section{RESULTS}

\section{Effect of canine prostate cancer cell conditioned medium on bone formation and resorption in vitro}

Ace-1, LuMa, and Probasco are known to metastasize to bone and induce different phenotypes of bone metastasis in $v i v o^{[5]}$. Ace-1 induces mixed osteoblastic/osteolytic bone metastasis, LuMa induces osteoblastic bone metastasis, and Probasco induces osteoblastic bone metastasis with abundant new bone formation. Calvaria were treated with CM from the three canine prostate cancer cells to determine if they would induce similar phenotypes in bone in vitro as compared to bone metastases in vivo. The ability of CM to induce bone formation was measured by uptake of the fluorescent dye, calcein. The results showed that Probasco $\mathrm{CM}$ induced bone formation with mineralization, while Ace-1 CM induced significantly less bone formation compared with controls. No difference in bone formation was seen between LuMa CM-treated calvaria and controls (calvaria treated with medium alone) [Figure $1 \mathrm{~A}$ and $\mathrm{B}$ ]. 
Table 1. Primers used for $\mathrm{PPT}-\mathrm{PCR}$ with mouse calvaria treated with control media or conditioned media collected from canine PCa cell lines

\begin{tabular}{|c|c|c|}
\hline Gene & Forward primer $\left(5^{\prime}-3^{\prime}\right)$ & Reverse primer $\left(5^{\prime}-3^{\prime}\right)$ \\
\hline$U B C$ & CGTCGAGCCCAGTGTTACCACCAAGAAGG & CCCCCATCACACCCAAGAACAAGCACAAG \\
\hline$R \cup N X 2$ & CGACAGTCCCAACTTCCTGT & TACCTCTCCGAGGGCTACAA \\
\hline OSX & ATGGCGTCCTCTCTGCTTG & ATGGCGTCCTCTCTGCTTG \\
\hline$A L P L$ & GGGCAATGAGGTCACATCCA & GTGGTTCACCCGAGTGGTAG \\
\hline RANKL & ACACCTCACCATCAATGCT & CTTAACGTCATGTTAGAGATCTTGG \\
\hline OPG & AGCTGCTGAAGCTGTGGAA & TCGAGTGGCCGAGAT \\
\hline OCN & CTCACAGATGCCAAGCCC & CCAAGGTAGCGCCGGAGTCT \\
\hline IBSP & CGGCGATAGTTCCGAAGAGG & GCTAGCTGTTACACCCGAGAG \\
\hline PTHTR & GGCAGCTGGGAGGTGGTT & CGAGTCTCATTGGTCATGAACTTG \\
\hline$O M D$ & GGTTGTGCTAAGGAATGCTTCT & TGAAGGTTGAGTTGCTGAATGTG \\
\hline ON & GTGGAAATGGGAGAATTTGAGGA & CTCACACACCTTGCCATGTTT \\
\hline MMP9 & CATTCGCGTGGATAAGGAGT & ATTTTGGAAACTCACACGCC \\
\hline CTSK & CTTCCAATACGTGCAGCAGA & ССTCTGCATTTAGCTGCCTT \\
\hline
\end{tabular}

PCa: Prostate cancer.

ALP activity was detected in protein extracted from PCa CM-treated calvaria. Consistent with bone mineralization, calvaria treated with Ace-1 CM showed the lowest ALP activity, there was no difference between control and LuMa CM-treated calvaria, and there was a marked increase in ALP activity in Probasco CM-treated calvaria [Figure 1C].

The effect of CM on bone resorption by osteoclasts was measured. Calvaria were treated with CM or control for 2 days. Calcium concentration in culture media was measured on day 1 and day 2. Ace-1 and LuMa CM increased bone resorption (greatest for Ace-1 CM) resulting in more calcium released into the medium compared to control, while there was no difference in bone resorption between Probasco CM-treated calvaria and control [Figure 1D]. These data demonstrated that CM from three canine prostate cancer cells induces different degrees of bone formation and resorption in vitro similar to the in vivo phenotype of bone metastases.

\section{Quantitative reverse-transcription polymerase chain reaction of CM-treated calvaria in vitro}

The expression of 12 bone-related genes including, an osteoblastogenesis maker (RUNX2), osteoblast differentiation-to-maturation makers (OSX, ALPL, RANKL, OPG, OCN, IBSP), osteoblast mineralization makers ( $P T H_{1} R, O M D$, and $\left.O N\right)$, and osteoclast-related genes (MMP9 and CTSK) was measured by quantitative reverse-transcription polymerase chain reaction in calvaria treated with $\mathrm{CM}$ or control for 3 days in order to investigate the potential mechanisms by which CM induced bone formation or resorption. Ace-1 CM upregulated the mRNA expression of RANKL over 60-fold, the expression of OPG 1.2-fold, and the expression of $M M P 9$ [Figure 2A]. This increased the ratio of RANKL/OPG mRNA over 50-fold in treated calvaria, which is consistent with increased in vitro bone resorption and the observed increase of calcium in the media released from Ace-1 CM-treated calvaria. In addition, the mRNA expression level of RUNX2, OSX, ALPL, OCN, IBSP, PTH1R, OMD, and ON was decreased markedly in Ace-1 CM-treated calvaria compared to controls, which was consistent with the result that Ace-1 CM had the least ability to stimulate bone mineralization in calvaria in vitro. In LuMa CM-treated calvaria, the relative mRNA expression levels of $O S X, A L P L, O C N, P T H 1 R$, and $O N$ were downregulated compared to controls [Figure 2B]. In Probasco CM-treated calvaria, the expression of RANKL, MMP9, and CTSK was significantly increased, which may be related to the increased bone turnover induced by Probasco CM [Figure $2 \mathrm{C}$ ]. 
A

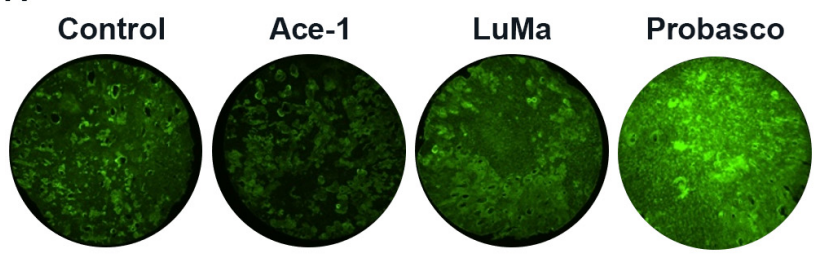

B

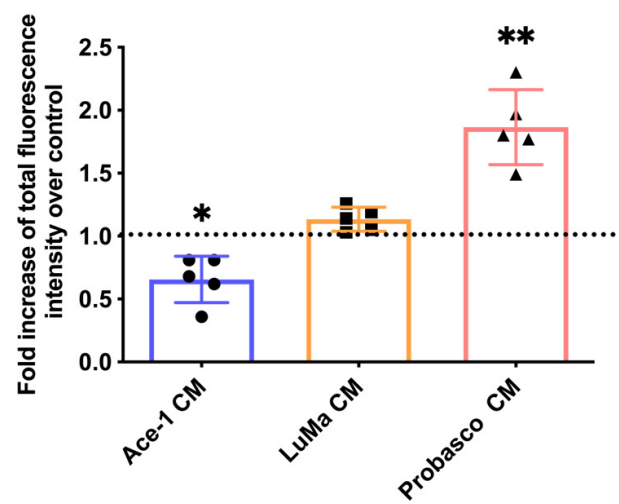

C

ALP activity
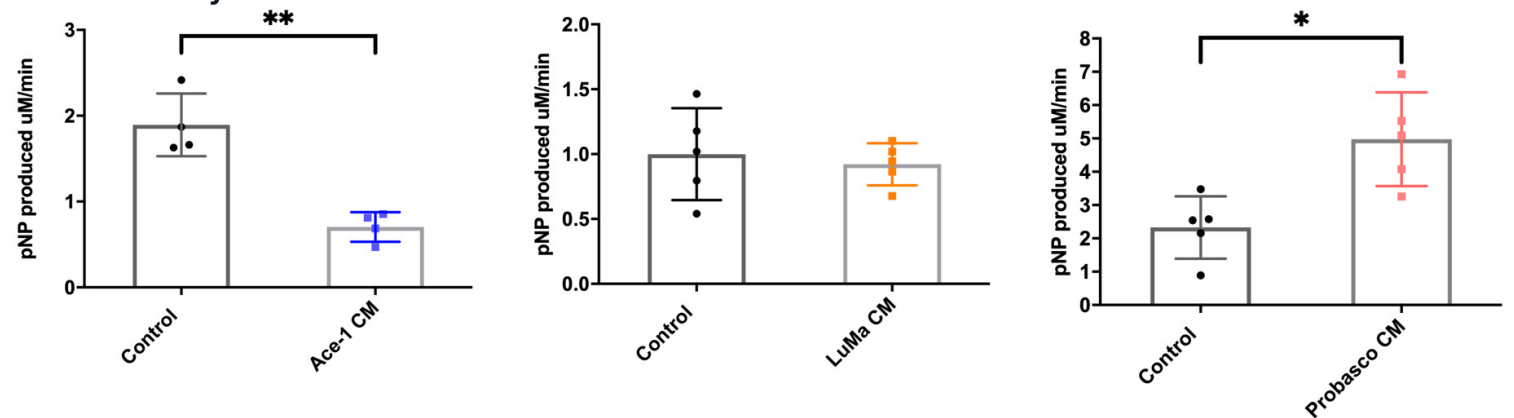

D Calcium concentration
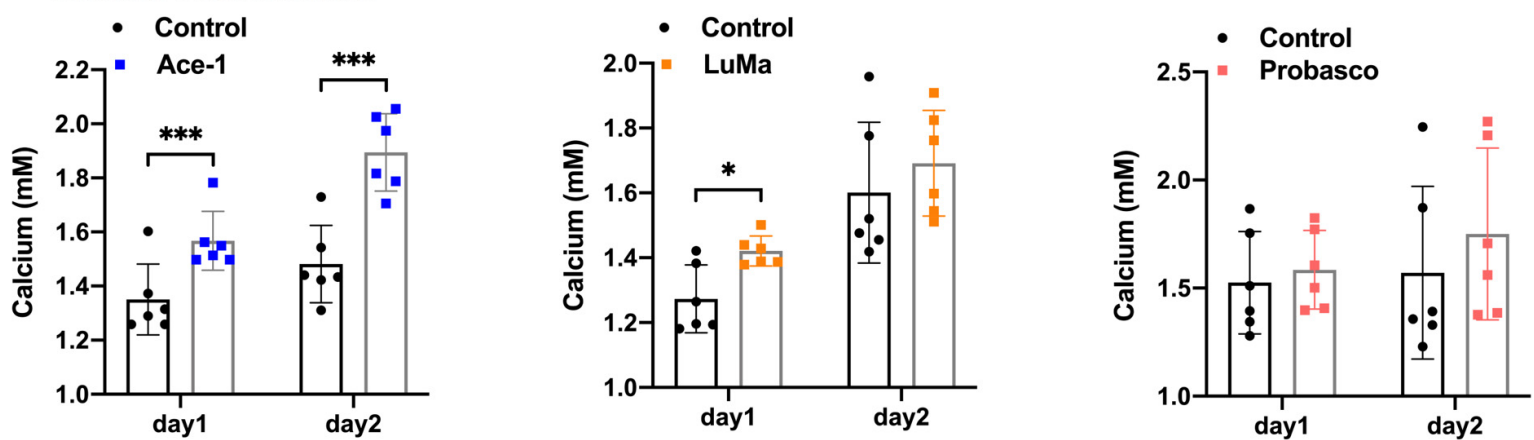

Figure 1. Effect of canine prostatic cancer cell conditioned media (CM) on bone formation and bone resorption in vitro. (A) Bone formation was visualized by calcein labeling of mineralization. Representative images of $1.5 \mathrm{~mm}$ calvarial disks (parietal bone). (B) The total fluorescence intensity was quantified and the fold increase over control was measured (5 pairs for each). (C) ALP activity in control calvaria and calvaria treated with canine PCa CM. (D) Amount of calcium present in the media for 2 days of culture. Data were expressed as mean $\pm \mathrm{SD}$. Significant differences were marked with asterisks $\left({ }^{\star} P<0.05,{ }^{\star \star} P<0.01,{ }^{\star \star \star} P<0.001\right)$. CM: Conditioned medium; ALP: alkaline phosphatase.

In addition, the mRNA expression of the genes was measured in LuMa or Probasco CM-treated calvaria and directly compared to Ace-1 CM-treated calvaria (as control) in order to investigate the different effect of the prostate cancer cell lines on calvaria. The relative mRNA expression level of RUNX2, OSX, ALPL, $O C N, P T H 1 R$, and $O M D$ was significantly increased, RANKL was markedly decreased, and there was no change in the expression of OPG in both Probasco and LuMa CM-treated calvaria compared to Ace-1 CM. The expression of IBSP was significantly increased in Probasco CM-treated calvaria [Figure 2D and E]. The results demonstrated that osteoblastic cell lines, LuMa and Probasco, induced bone formation by the stimulation of maturation of osteoblasts to form mineralized bone, and the inhibited osteoclastogenesis in vitro. 

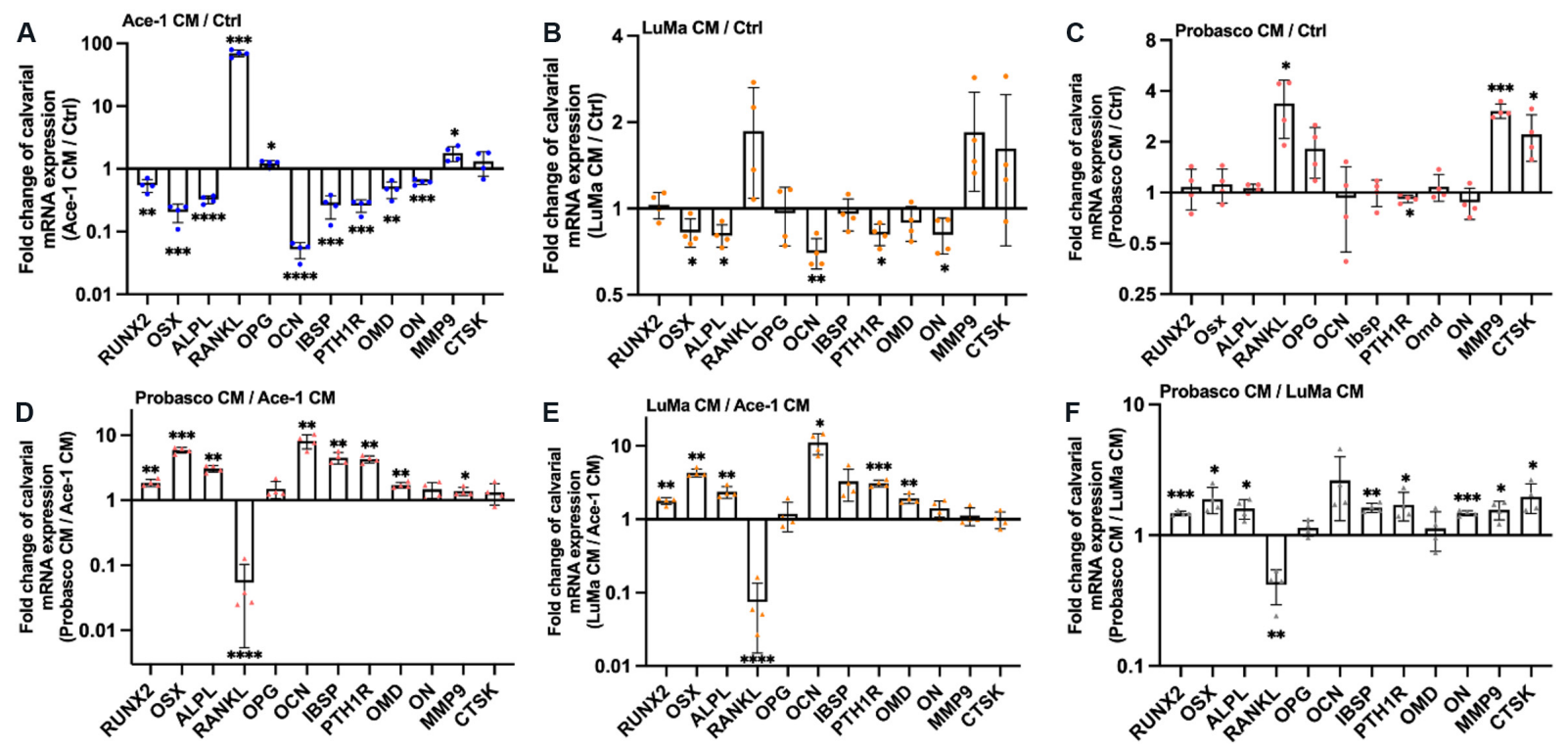

Figure 2. Expression of bone-related genes in calvaria treated with $C M$ or control. Graphs represent the fold changes of mRNA expression of RUNX2, OSX, ALPL, RANKL, OPG, OCN, IBSP, PTH1R, OMD, ON, MMP9, and CTSK in (A) Ace-1 CM-treated calvaria relative to control; (B) LuMa CM-treated calvaria relative to control; (C) Probasco CM-treated calvaria relative to control; (D) Probasco CMtreated calvaria relative to Ace-1 CM-treated calvaria; (E) LuMa CM-treated calvaria relative to Ace-1 CM-treated calvaria; and (F) Probasco CM-treated calvaria relative to LuMa CM-treated calvaria. Data were expressed as mean \pm SD. Significant differences were marked with asterisks $\left({ }^{\star} P<0.05,{ }^{\star \star} P<0.01,{ }^{\star \star \star} P<0.001,{ }^{\star \star \star \star} P<0.0001\right)$. Note differences in the scales of the $Y$ axes. $C M$ : Conditioned medium.

Furthermore, the comparison of the mRNA expression between Probasco CM-treated calvaria and LuMa CM-treated calvaria revealed that Probasco had greater osteoblastic ability compared to LuMa, which is consistent with the observed bone mineralization on calvaria induced by Probasco CM [Figure 2F].

\section{Effect of Probasco CM on bone formation in mouse calvaria defects}

Probasco CM improved the healing response of calvarial defects when compared to control calvaria [Figure 3A]. The repaired area in the defect of Probasco CM-treated calvaria was increased 2-fold compared to control [Figure 3B], which demonstrated that Probasco CM stimulated bone formation in bone defects in vitro.

\section{Probasco $\mathrm{CM}$ increased bone formation through bone remodeling and modeling}

Bone shape and structure are a result of bone modeling (bone formation by osteoblasts independent of osteoclasts) and bone remodeling (the removal of mineralized bone by osteoclasts followed by the formation of bone matrix by osteoblasts that subsequently becomes mineralized). To determine if bone modeling was involved in Probasco CM-induced bone formation, bone remodeling was inhibited by blocking osteoclast formation and function with the bisphosphonate ${ }^{[12]}$, zoledronic acid (ZOL; Zometa ${ }^{\mathrm{TM}}$ ). Inhibition of bone remodeling greatly decreased bone formation in the control group; however, Probasco $\mathrm{CM}$ was still able to induce bone formation [Figure $4 \mathrm{~A}$ and $\mathrm{B}$ ], which revealed that both remodeling and modeling were involved in Probasco CM-induced bone formation. Additionally, calvaria treated with Probasco CM were evaluated by histology. Frozen sections and H\&E staining showed that calcein was deposited in the bone matrix and marked periosteal thickening was induced by Probasco CM [Figure 4C and D]. 
A

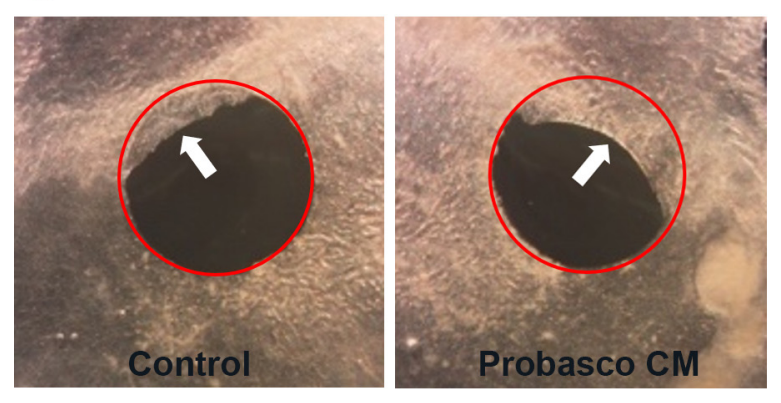

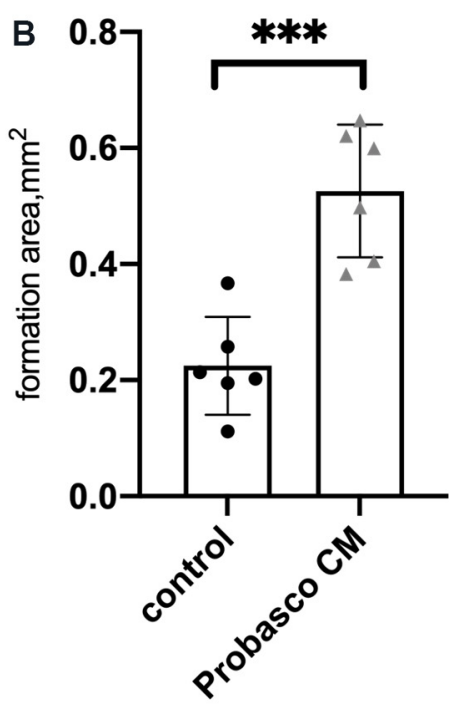

Figure 3. Effect of Probasco CM on mouse calvarial defects. (A) Representative microscopic images. Calvaria with defects (red circle showing the initial $1.5 \mathrm{~mm}$ round artificial defect before treatment) incubated with only BGJb had less repaired area (white arrow) compared to calvaria treated with Probasco CM. (B) Repaired area was quantified. Data were expressed as mean \pm SD. Significant differences were marked with asterisks $\left.{ }^{\star \star \star} P<0.001\right)$. CM: Conditioned medium.

\section{A}

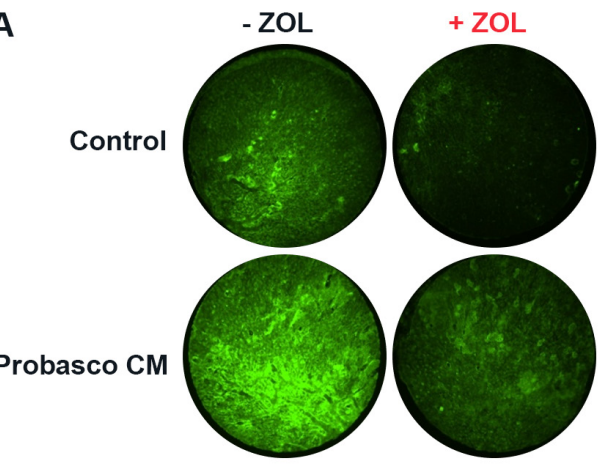

C

Contro

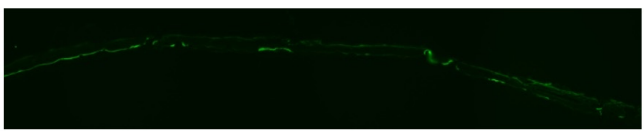

Probasco CM

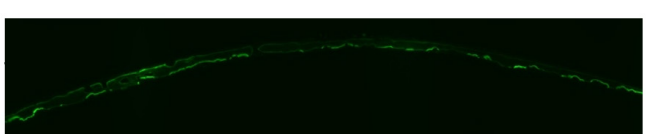

B

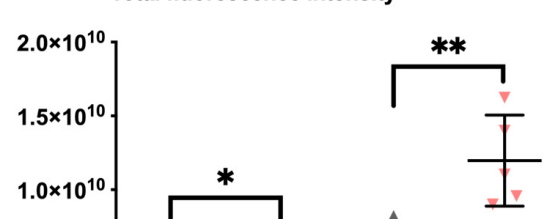

Vehicle

Probaco $\mathrm{CM}(50 \%)$

Probaco $\mathrm{CM}$

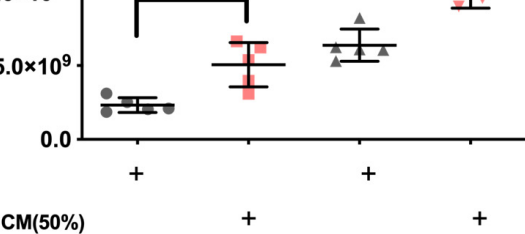

D

Control

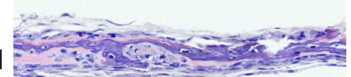

Probasco CM

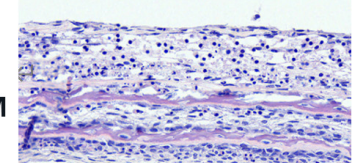

Figure 4. Probasco $\mathrm{CM}$ increased bone formation through bone remodeling and modeling. Calvaria were divided into two groups. One group was pre-treated with zoledronic acid $\left(Z O L, 10^{-5} \mathrm{M}\right)$ and the other group was treated with vehicle overnight. In each pair of calvaria, a half calvarium was treated with Probasco CM or control medium. (A) Representative images of $1.5 \mathrm{~mm}$ calvarial disks taken from the parietal bone. Probasco CM increased bone formation and mineralization (calcein uptake) compared to control. (B) The total fluorescence intensity was quantified. Data were expressed as mean \pm SD and analyzed by paired $t$-test. Significant differences were marked with asterisks $\left({ }^{\star} P<0.05,{ }^{\star \star} P<0.01\right)$. (C) Frozen section of calvaria treated with control media or Probasco CM. Calcein was deposited in mineralized bone matrix on both surfaces of the calvaria. (D) H\&E-stained section of calvaria. There was marked periosteal thickening and new bone formation caused by Probasco CM. CM: Conditioned medium.

\section{Stimulation of bone formation by Probasco CM was dependent on the WNT signaling pathway} Our laboratory has shown that blocking WNT action with DKK-1 inhibited the osteoblastic phenotype 
induced by Ace- 1 in nude mice ${ }^{[11]}$. To test whether the stimulation of bone formation by Probasco CM also depended on WNT signaling pathway, calvaria were treated with CM from either Probasco+DKK-1 or Probasco cells. Secretion of human DKK-1 by Probasco+DKK-1 cells was confirmed by ELISA. CM collected from Probasco cells had no detectable DKK-1 secretion while Probasco+DKK-1 CM contained approximately $8 \mathrm{ng} / \mathrm{mL}$ after $24 \mathrm{~h}$ of culture [Figure 5A]. Probasco+DKK-1 CM strongly inhibited bone mineralization [Figure $5 \mathrm{~B}$ and $\mathrm{C}$ ]. Calvaria pre-treated with $\mathrm{ZOL}$, in which osteoclast activity and bone remolding were blocked by ZOL, indicated that Probasco+DKK-1 inhibited bone modeling induced by Probasco CM. Frozen sections confirmed that less calcein was deposited in Probasco+DKK-1 CM-treated calvaria matrix compared with the calvaria treated by Probasco CM [Figure 5D]. In addition, there was a marked decrease in ALP activity in Probasco+DKK-1-treated calvaria [Figure 5E]. The mRNA expression of osteoblast-related genes was measured in calvaria. The level of OCN was significantly decreased in Probasco+DKK-1 CM-treated calvaria compared to calvaria treated with Probasco CM [Figure 5F]. OCN is a marker of mature osteoblasts. Osteocalcin is secreted by osteoblasts into the bone micro-environment and regulates extracellular matrix mineralization ${ }^{[16]}$. The reduced expression of $O C N$ supports the observed decrease in mineralization caused by Probasco+DKK-1 CM. In addition, the downregulation of $O C N$ in Probasco+DKK-1 CM-treated calvaria reveals that blocking WNT signaling pathway by DKK-1 may inhibit osteoblast differentiation in vitro. These results showed that inhibition of the WNT signaling pathway by DKK-1 significantly decreased bone formation induced by Probasco CM.

\section{DISCUSSION}

We successfully developed a novel method to measure bone formation and mineralization in vitro. In vitro bone resorption assays have been used for decades to measure osteoclastic bone resorption; however, it has been particularly challenging to measure bone formation in vitro. The use of the fluorescent dye, calcein, enabled increased sensitivity for the measurement and quantitation of bone mineralization in vitro. This newly described assay will be helpful for mechanistically investigating the pathogenesis of bone formation induced by prostate cancer.

We showed that conditioned media from canine prostate cancer cells induced bone resorption and formation in vitro that correlated with the in vivo phenotype of bone metastases associated with each cell line. Ace-1 CM mainly induced bone resorption, while Probasco CM significantly stimulated bone formation by both bone modeling and remolding in mouse calvaria. Additionally, we showed that inhibition of WNT activity by DKK-1 blocked the osteoblastic activity in Probasco cells.

Animal models are critical for investigating the pathogenesis of prostate cancer-induced bone metastasis. Currently, a few animal models are available that closely mimic the osteoblastic property of human prostate cancer. Human LNCap subline C4-2B and the LuCaP23.1 tumor cell lines are two most commonly used human cell lines that have an osteoblastic phenotype; however, both of them induce mixed bone tumors as they stimulate significant bone lysis as well as formation ${ }^{[17,18]}$. As for murine models, most transgenic mouse models do not develop bone metastases, and bone xenograft models of prostate cancer often induce mixed or osteolytic bone tumors ${ }^{[5]}$. In the present study, we used three canine cell lines (Ace-1, LuMa and Probasco). The ability of the Probasco cells to induce osteoblastic bone formation both in vivo and in vitro, makes it especially valuable for investigating the mechanisms of osteoblastic metastasis in prostate cancer. In addition, by comparing the genetic expression profile of osteoblastic Probasco cells with osteolytic Ace-1 cells, it may uncover critical pathways that contribute to the osteoblastic phenotype in prostate cancer.

Normal bone development consists of two processes, bone remodeling, defined by the sequential actions of bone resorption and bone formation, and bone modeling, characterized by the independent actions of 


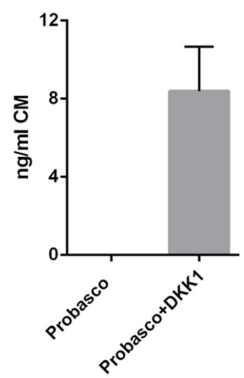

D

Probasco+DKK-1 CM

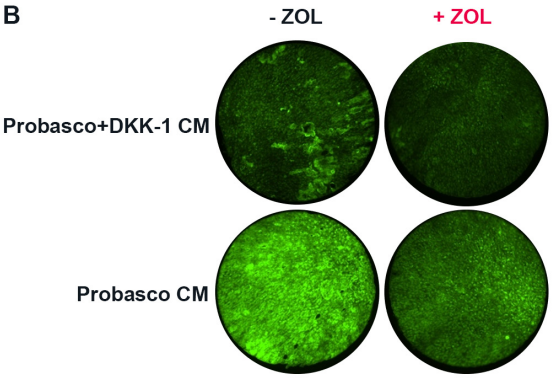

E ALP activity

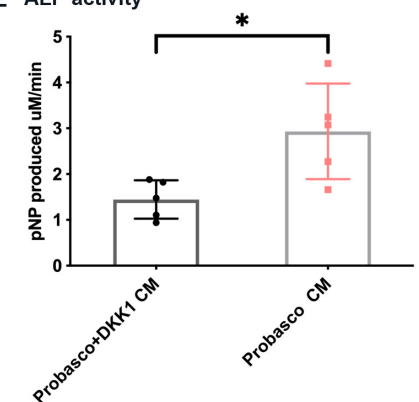

C

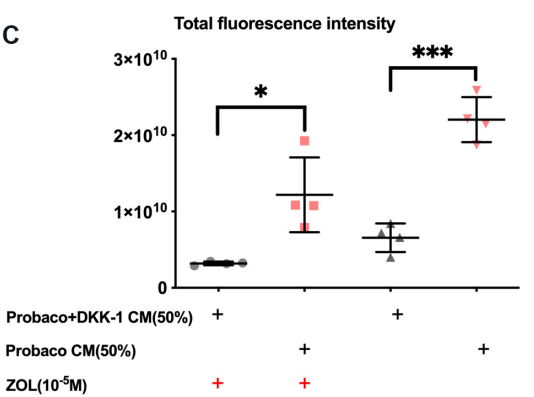

$\mathbf{F}$

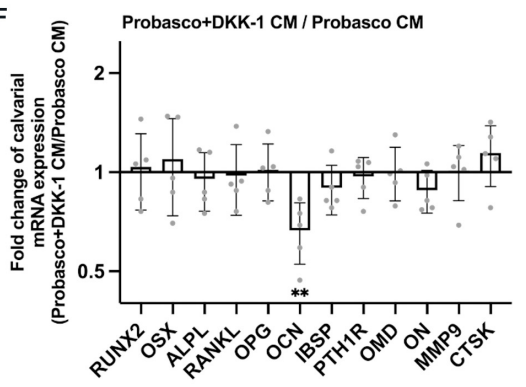

Figure 5. Stimulation of bone formation by Probasco CM was dependent on the WNT signaling pathway. (A) Concentration of secreted human DKK-1 in Probasco CM and Probasco+DKK-1 CM. (B) Representative images of $1.5 \mathrm{~mm}$ calvarial disks from parietal bone. Probasco+DKK-1 significantly decreased bone formation. (C) The total fluorescence intensity was quantified. (D) Frozen section of calvaria treated Probasco+DKK-1 CM or Probasco CM. More calcein was deposited in the bone matrix. (E) ALP activity in calvaria treated with Probasco CM and calvaria treated with Probasco+DKK-1 CM. (F) The mRNA expression of bone-related genes in Probasco+DKK-1 CM-treated calvaria compared to Probasco CM-treated calvaria. Data were expressed as mean \pm SD and analyzed by paired $t$-test. Significant differences were marked with asterisks $\left.\left({ }^{\star} P<0.05,{ }^{* \star} P<0.01\right),{ }^{\star \star \star} P<0.001\right)$.

osteoblasts and osteoclasts ${ }^{[19]}$. Histologic assessment of PCa-induced bone metastases demonstrates that the radiodense bone metastases were largely composed of woven bone which has less structural strength compared to lamellar bone ${ }^{[20]}$. The formation of woven bone is often caused by bone modeling since the bone formation occurs rapidly and is not dependent on initial osteoclastic bone resorption. Our in vitro results showed that Probasco CM induced osteoblastic bone formation in part by bone modeling since Probasco CM induced bone formation in ZOL pretreated-calvaria.

WNTs are a large family of regulatory proteins that direct embryological development and promote bone growth $^{[21]}$, acting through both canonical and non-canonical signaling pathways. Proteins secreted by prostate cancer cells exert their function via autocrine and paracrine actions, and result in the stimulation of PCa growth and osteoblast activity ${ }^{[10]}$. It has been reported that inhibition of WNT activity switched the mixed osteoblastic/osteolytic C4-2B cell line to a highly osteolytic cell line $e^{[\rho]}$. Also, PCa cells treated with WNT3A or WNT5A promoted the differentiation of osteoblasts through the canonical and non-canonical pathways in a BMP-dependent and -independent manner, which demonstrated crosstalk between WNTs and BMPs in PCa-induced osteoblast differentiation ${ }^{[2]}$. Our in vitro results, in agreement with these findings, showed that increasing DKK-1 expression in Probasco blocked WNT-induced osteoblastic activity. Based on RNA-seq analysis of canine PCa cell lines (unpublished data), we found that several WNTs proteins (WNT3, WNT5A, and WNT7B) as well as BMPs (BMP-2, BMP-4, and BMP-7) are expressed in Probasco cells. It will be promising to further investigate if both WNTs and BMPs play a role as mediators of Probasco-induced osteoblastic activity. 
ALP is an important enzyme in bone formation, and it is highly expressed in mineralized bone cells ${ }^{[23]}$. While the measurement of ALP expression is well developed and is a reliable indication of the osteoblastic phenotype, the regulation of ALP expression and function in the mineralization of bone is less clear. The regulation of ALP expression is mainly controlled by BMP/RUNX2/Osterix system, and the WNT signaling cascade is also involved in the process ${ }^{[24,25]}$. The capacity of BMP-2 to induce ALP relies on WNT expression and the WNT/LRP5 signaling cascade. BMP-2 mediates extracellular matrix mineralization by osteoblasts partly through the induction of a WNT autocrine/paracrine loop ${ }^{[26]}$. BMP-2 is highly expressed in Probasco cells compared to Ace-1 cells ${ }^{[7]}$. Secreted BMP-2 in Probasco CM could be an important factor stimulating bone mineralization and the markedly increased ALP activity in CM treated-calvaria. In addition, decreased ALP activity in Probasco+DKK-1 CM treated calvaria compared to calvaria treated with Probasco CM suggests that the regulation of BMP-2 on ALP is dependent on WNT signaling. qPT-PCR analysis revealed no difference in the mRNA expression of ALPL in Probasco CM-treated calvaria compared to control, which indicates that the induction of ALP activity may be regulated at the post-translational level.

Runx2 and osterix regulate the differentiation of osteoblasts. Runx2 or osterix-deficient mice have a complete absence of bone formation and osteoblasts, indicating that these two transcription factors are essential for bone development and osteoblastogenesis ${ }^{[27]}$. BMP-2 can markedly upregulate the expression of RUNX2 through the activation of Smad signaling ${ }^{[28,29]}$, and the expression of OSX is also under the control of BMP- $2^{[30]}$. By comparing calvaria treated with CM from osteoblastic cell lines (LuMa and Probasco) to calvaria treated with CM from osteolytic cell line (Ace-1), the mRNA expression of RUNX2 and OSX was significantly upregulated in both LuMa and Probasco CM-treated calvaria. Since there is high expression of BMP-2 in LuMa and Probasco cell lines compared to Ace-1 cells, and both RUNX2 and OSX are target genes of BMP-2, it's speculated that BMP-2 plays a role in canine PCa-induced bone formation.

Bone integrin-binding sialoprotein (IBSP) is the second major sialoprotein in bone. IBSP is synthesized by osteoblasts and its expression is tightly associated to mineralization. IBSP expression marks a late stage of osteoblastic differentiation and an early stage of matrix mineralization ${ }^{[31]}$. The significant upregulation of IBSP was observed in Probasco CM-treated calvaria, but not in LuMa CM-treated calvaria compared to Ace-1 CM-treated calvaria, which may reflect that Probasco was more potent on the induction of mineralization compared to LuMa. Current studies on IBSP often focus on the role of IBSP in osteotropic malignancies, such as multiple myeloma, breast, prostate and lung cancer, and tumor progression and bone metastasis ${ }^{[32,33]}$. Little is known about factors secreted by these malignancies that induce the expression of IBSP in bone cells.

In conclusion, Probasco, an osteoblastic canine prostate cancer cell line, induces bone formation and mineralization in mouse calvaria in vitro by the stimulation of maturation and mineralization of osteoblasts, and the inhibition of osteoclastogenesis. Both bone modeling and remodeling are involved in Probascoinduced bone formation, and the induction depends on the WNT signaling pathway. The Probasco cell line provides a valuable and unique model for understanding the molecular mechanisms of prostate cancerinduced osteoblastic bone metastases.

\section{DECLARATIONS}

\section{Acknowledgements}

We thank the Histology Core Laboratory in the Department of Biomedical Science and Julie Buckley for sectioning and staining of tissue samples. 


\section{Authors' contributions}

Designed overall projects; manuscript preparation and edit: Rosol TJ, Yuan S

Performed experiments: Yuan S, Hellmann DE

Analyzed and interpreted data: Yuan S, Rosol TJ, Kantake N

Technical and material support: Kantake N, Elshafae SM

\section{Availability of data and materials}

The material and data that support the findings of this study are available from the corresponding author upon request.

\section{Financial supports and sponsorship}

This work was supported by the Heritage College of Osteopathic Medicine at Ohio University and a John J. Kopchick Fellowship Award to SY.

\section{Conflicts of interest}

All authors declared that there are no conflicts of interest.

\section{Ethical approval and consent to participate}

All mice used were housed in the animal facilities at Ohio University. Animal experiments were performed with the approval of the Institutional Animal Care and Use Committee (IACUC) (protocol number: 18-H030) and in accordance with the Ethical Guidelines for Animal Experimentation of Ohio University.

\section{Consent for publication}

Not applicable.

\section{Copyright}

(c) The Author(s) 2021.

\section{REFERENCES}

1. Logothetis CJ, Lin SH. Osteoblasts in prostate cancer metastasis to bone. Nat Rev Cancer 2005;5:21-8. DOI PubMed

2. Park SH, Keller ET, Shiozawa Y. Bone marrow microenvironment as a regulator and therapeutic target for prostate cancer bone metastasis. Calcif Tissue Int 2018;102:152-62. DOI PubMed PMC

3. Rosol TJ, Tannehill-Gregg SH, LeRoy BE, Mandl S, Contag CH. Animal models of bone metastasis. Cancer 2003;97:748-57. DOI PubMed

4. Keller JM, Schade GR, Ives K, et al. A novel canine model for prostate cancer. Prostate 2013;73:952-9. DOI PubMed

5. Simmons JK, Hildreth BE 3rd, Supsavhad W, et al. Animal models of bone metastasis. Vet Pathol 2015;52:827-41. DOI PubMed PMC

6. LeRoy BE, Thudi NK, Nadella MV, et al. New bone formation and osteolysis by a metastatic, highly invasive canine prostate carcinoma xenograft. Prostate 2006;66:1213-22. DOI PubMed

7. Simmons JK, Dirksen WP, Hildreth BE 3rd, et al. Canine prostate cancer cell line (Probasco) produces osteoblastic metastases in vivo. Prostate 2014;74:1251-65. DOI PubMed PMC

8. Elshafae SM, Dirksen WP, Alasonyalilar-Demirer A, et al. Canine prostatic cancer cell line (LuMa) with osteoblastic bone metastasis. Prostate 2020;80:698-714. DOI PubMed PMC

9. Hall CL, Bafico A, Dai J, Aaronson SA, Keller ET. Prostate cancer cells promote osteoblastic bone metastases through Wnts. Cancer Res 2005;65:7554-60. DOI PubMed

10. Murillo-Garzón V, Kypta R. WNT signalling in prostate cancer. Nat Rev Urol 2017;14:683-96. DOI PubMed

11. Thudi NK, Martin CK, Murahari S, et al. Dickkopf-1 (DKK-1) stimulated prostate cancer growth and metastasis and inhibited bone formation in osteoblastic bone metastases. Prostate 2011;71:615-25. DOI PubMed PMC

12. Thudi NK, Martin CK, Nadella MV, et al. Zoledronic acid decreased osteolysis but not bone metastasis in a nude mouse model of canine prostate cancer with mixed bone lesions. Prostate 2008;68:1116-25. DOI PubMed PMC

13. Hale LV, Ma YF, Santerre RF. Semi-quantitative fluorescence analysis of calcein binding as a measurement of in vitro mineralization. Calcif Tissue Int 2000;67:80-4. DOI PubMed

14. Dimai HP, Hall SL, Stilt-Coffing B, Farley JR. Skeletal response to dietary zinc in adult female mice. Calcif Tissue Int 1998;62:30915. DOI PubMed

15. Lorentz K. Improved determination of serum calcium with 2-cresolphthalein complexone. Clinica Chimica Acta 1982;126:327-34. 
DOI PubMed

16. Moser SC, van der Eerden BCJ. Osteocalcin-a versatile bone-derived hormone. Front Endocrinol (Lausanne) 2018;9:794. DOI PubMed PMC

17. Zhang J, Dai J, Qi Y, et al. Osteoprotegerin inhibits prostate cancer-induced osteoclastogenesis and prevents prostate tumor growth in the bone. J Clin Invest 2001;107:1235-44. DOI PubMed PMC

18. Corey E, Quinn JE, Bladou F, et al. Establishment and characterization of osseous prostate cancer models: intra-tibial injection of human prostate cancer cells. Prostate 2002;52:20-33. DOI PubMed

19. Langdahl B, Ferrari S, Dempster DW. Bone modeling and remodeling: potential as therapeutic targets for the treatment of osteoporosis. Ther Adv Musculoskelet Dis 2016;8:225-35. DOI PubMed PMC

20. Roudier MP, Morrissey C, True LD, Higano CS, Vessella RL, Ott SM. Histopathological assessment of prostate cancer bone osteoblastic metastases. J Urol 2008;180:1154-60. DOI PubMed PMC

21. Regard JB, Zhong Z, Williams BO, Yang Y. Wnt signaling in bone development and disease: making stronger bone with Wnts. Cold Spring Harb Perspect Biol 2012;4:a007997. DOI PubMed PMC

22. Dai J, Hall CL, Escara-Wilke J, Mizokami A, Keller JM, Keller ET. Prostate cancer induces bone metastasis through Wnt-induced bone morphogenetic protein-dependent and independent mechanisms. Cancer Res 2008;68:5785-94. DOI PubMed PMC

23. Orimo H, Shimada T. The role of tissue-nonspecific alkaline phosphatase in the phosphate-induced activation of alkaline phosphatase and mineralization in SaOS-2 human osteoblast-like cells. Mol Cell Biochem 2008;315:51-60. DOI PubMed

24. Gaur T, Lengner CJ, Hovhannisyan H, et al. Canonical WNT signaling promotes osteogenesis by directly stimulating Runx2 gene expression. J Biol Chem 2005;280:33132-40. DOI PubMed

25. Gaur T, Rich L, Lengner CJ, et al. Secreted frizzled related protein 1 regulates Wnt signaling for BMP2 induced chondrocyte differentiation. J Cell Physiol 2006;208:87-96. DOI PubMed

26. Rawadi G, Vayssière B, Dunn F, Baron R, Roman-Roman S. BMP-2 controls alkaline phosphatase expression and osteoblast mineralization by a Wnt autocrine loop. J Bone Miner Res 2003;18:1842-53. DOI PubMed

27. Pérez-Campo FM, Santurtún A, García-Ibarbia C, et al. Osterix and RUNX2 are transcriptional regulators of sclerostin in human bone. Calcif Tissue Int 2016;99:302-9. DOI PubMed

28. Nishimura R, Hata K, Harris S, Ikeda F, Yoneda T. Core-binding factor $\alpha 1$ (Cbfa1) induces osteoblastic differentiation of $\mathrm{C} 2 \mathrm{C} 12$ cells without interactions with Smad1 and Smad5. Bone 2002;31:303-12. DOI PubMed

29. Alliston T, Choy L, Ducy P, Karsenty G, Derynck R. TGF-beta-induced repression of CBFA1 by Smad3 decreases cbfa1 and osteocalcin expression and inhibits osteoblast differentiation. EMBO J 2001;20:2254-72. DOI PubMed PMC

30. Nakashima K, Zhou X, Kunkel G, et al. The novel zinc finger-containing transcription factor osterix is required for osteoblast differentiation and bone formation. Cell 2002;108:17-29. DOI PubMed

31. Malaval L, Wade-Guéye NM, Boudiffa M, et al. Bone sialoprotein plays a functional role in bone formation and osteoclastogenesis. $J$ Exp Med 2008;205:1145-53. DOI PubMed PMC

32. Kruger TE, Miller AH, Godwin AK, Wang J. Bone sialoprotein and osteopontin in bone metastasis of osteotropic cancers. Crit Rev Oncol Hematol 2014;89:330-41. DOI PubMed PMC

33. Zhang L, Hou X, Lu S, et al. Predictive significance of bone sialoprotein and osteopontin for bone metastases in resected Chinese nonsmall-cell lung cancer patients: a large cohort retrospective study. Lung Cancer 2010;67:114-9. DOI PubMed 